\title{
Profitable Cropping Systems for Southern Telangana Zone of Telangana State, India
}

\author{
Ch. Pragathi Kumari*, S. Sridevi and M. Goverdhan \\ AICRP on Integrated Farming Systems, PJTSAU, Rajendranagar, Hyderabad-30, \\ Telangana, India \\ *Corresponding author
}

\section{A B S T R A C T}

\section{Keywords}

Cropping systems, System productivity, Profitability, Nutrient removal, Soil fertility

Article Info

Accepted:

20 December 2017

Available Online:

10 January 2018
A field experiment was conducted during 2016-17 at AICRP on Integrated Farming Systems, PJTSAU to identify bio-intensive complementary cropping systems under I.D conditions for light textured soils of Southern Telangana Zone. Amongst the twelve cropping systems tested, cotton + green gram (1:2) - maize for green cobs

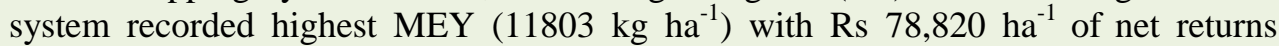
followed by $B t$ cotton + soybean (1:3) - sesame + groundnut (2:4) system $(10155 \mathrm{~kg}$ $\left.\mathrm{ha}^{-1}\right)$. Bt Cotton + green gram $(1: 2)-$ maize for green cobs system removed significantly higher nitrogen $\left(189.2 \mathrm{~kg} \mathrm{ha}^{-1}\right)$ and potassium $\left(158.4 \mathrm{~kg} \mathrm{ha}^{-1}\right)$. Removal of phosphorus was significantly more by all maize based cropping systems. The Post harvest soil analysis after kharif and also at the end of different crops/cropping systems did not show any remarkable changes in $\mathrm{pH}, \mathrm{EC}$, organic carbon, available potassium and phosphorus.

\section{Introduction}

Crop diversification has been recognized as an effective strategy for achieving the objectives of food security, nutrition security, income growth, poverty alleviation, employment generation, judicious use of land and water resources, sustainable agricultural development and environmental improvement (Hedge et al., 2003). In the era of shrinking resource base of land, water and energy, resource use efficiency is an important aspect for considering the sustainability of a cropping system (Yadav, 2002). Cotton-maize, maizesunflower is a widely practiced predominant cropping systems in the Southern Telangana Zone. Both being exhaustive, non-leguminous in nature the viability and sustainability of system is a matter of concern especially in the context of light textured soils of the region.

Hence identification of bio intensive complimentary cropping systems by inclusion of more hardy cereals and pulse crops in the system which are ecologically sustainable and more viable is very pertinent. The current study mainly aimed at evolving alternative cropping systems with higher productivity and profitability over the widely adopted of maize - sunflower, cotton - maize cropping systems. 


\section{Materials and Methods}

The study was conducted at research farm of All India Coordinated Research Project on Integrated Farming Systems, Professor Jayashankar Telangana Sate Agricultural University, Rajendranagr during 2016-17. The soil of the experimental field was a red chalka soil. The experiment was laid out with twelve cropping systems as treatments in Randomized Block Design (RBD) with three replications. The twelve combinations of biointensive complimentary cropping systems tested during kharif and rabi seasons were, $\mathrm{T}_{1}$ : Maize - sunflower (check), $\mathrm{T}_{2}$ : Pearl millet + soybean-potato, $\mathrm{T}_{3}$ : Maize-groundnut, $\mathrm{T}_{4}$ : Pearl millet + soybean $(3: 2)$ - sunflower + groundnut (2:3), $\mathrm{T}_{5}$ : Maize + soybean $(2: 3)-$ potato, $\mathrm{T}_{6}: B t$ cotton + soybean $(1: 3)-$ sesame + groundnut, $\mathrm{T}_{7}$ : Maize (Flatbed) + soybean (Raised Bed) - castor $(\mathrm{F})+$ greengram $(\mathrm{RB})$ (2:3), $\mathrm{T}_{8}: B t$ cotton + greengram (1:3) - pearl millet, $\mathrm{T}_{9}$ : Soybean - potato, $\mathrm{T}_{10}: B t$ cotton + green gram (1:2) -sesame, $\mathrm{T}_{11}$ : Pearl millet groundnut, $\mathrm{T}_{12}$ : Bt Cotton + green gram (1:2) -maize for green cobs.

All the kharif crops were sown on 2.07.2016 and the following sequence crops during rabi were taken up as and when the preceding kharif crops were harvested in the respective plots. Economic yield and stover/straw/stalk yield were recorded individually for all the crops in cropping systems.

For comparison of different crop sequences, the yields of all the crops were converted in to maize equivalent yield on price basis. Nutrient removal by different cropping sequences was worked out by estimating the nutrient concentrations (N, P and $\mathrm{K}$ ) in grain and straw of crops. To understand the impact of various cropping systems on soil fertility, post-harvest soil was analysed for $\mathrm{pH}, \mathrm{EC}$, organic carbon and available $\mathrm{N}, \mathrm{P}$ and $\mathrm{K}$ status by following the standard procedures (Jackson, 1973).

\section{Results and Discussion}

\section{Productivity and economics of crops and cropping systems}

The performance of different crops in terms of maize equivalent yield (MEY) during kharif, 2016 indicated that $B t$ cotton intercropped with soybean at 1:3 ratio gave significantly higher maize equivalent yield (7516 $\left.\mathrm{kg} \mathrm{ha}^{-1}\right)$ over other crops or cropping systems (Table 1 and 2). However it was found to be at par with $B t$ cotton intercropped with greengram at 1:3 (7474 $\mathrm{kg} \mathrm{ha}^{-1}$ ) or $B t$ cotton intercropped with greengram in 1:2 row ratio $\left(6945 \mathrm{~kg} \mathrm{ha}^{-1}\right)$.

The lowest maize equivalent yield was recorded with pearlmillet intercropped with soybean in 3:2 row ratio $\left(1742 \mathrm{~kg} \mathrm{ha}^{-1}\right)$. Sreerekha et al., (2010) reported that cotton hybrid, Bunny and variety, Narsimha inter cropped with soybean recorded 28 and 29 per cent more seed cotton yield, respectively, over corresponding sole crops. Due to higher price of greengram, net returns from $B t$ cotton intercropped with greengram in 1:3 row ratio system were higher (Rs $48676 \mathrm{ha}^{-1}$ ) followed by $B t$ Cotton intercropped with soybean at 1:3 row ratio (Rs $46345 \mathrm{ha}^{-1}$ ) and $B t$ cotton intercropped with greengram at 1:2 (Rs. 43425 $\mathrm{ha}^{-1}$ ). Similar maximum net returns (Rs 61604) was observed in cotton + mung intercropping than all other paired row cotton with intercrops (CICR, 2009-10).

During rabi 2016, potato crop raised after soybean, recorded significantly highest MEY of $5260 \mathrm{~kg} \mathrm{ha}^{-1}$ over other tested crops or cropping systems. It was closely followed by maize grown for green cobs $\left(5173 \mathrm{~kg} \mathrm{ha}^{-1}\right)$ and potato $\left(4852 \mathrm{~kg} \mathrm{ha}^{-1}\right)$ raised after pearlmillet + soybean system. Potato being nontraditional crop provides excellent opportunities in raising the income of the farmers as it has capacity to yield 5-10 times more than cereals, pulses or oilseeds. 
Table.1 Performance of crops under bio-intensive complementary cropping systems

\begin{tabular}{|c|c|c|c|c|c|c|c|c|c|c|c|c|}
\hline \multirow{2}{*}{\multicolumn{2}{|c|}{$\begin{array}{l}\text { Treatments } \\
\text { Kharif-Rabi }\end{array}$}} & \multicolumn{2}{|c|}{ Kharif (2016) } & \multicolumn{2}{|c|}{ Rabi (2016-17) } & \multicolumn{4}{|c|}{ Maize Equivalent Yield $\left(\mathrm{kg} \mathrm{ha}^{-1}\right)$} & \multirow{2}{*}{\multicolumn{3}{|c|}{$\begin{array}{c}\text { Productivity } \\
\text { (MEY -kg ha }^{-1} \text { ) }\end{array}$}} \\
\hline & & \multirow{2}{*}{$\begin{array}{c}\text { Grain yield } \\
\left(\mathrm{kg} \mathrm{ha}^{-1}\right)\end{array}$} & \multirow{2}{*}{$\begin{array}{c}\text { Straw/ } \\
\text { Stover } \\
\text { yield } \\
\left(\mathbf{k g ~ h a}^{-1}\right)\end{array}$} & \multirow{2}{*}{$\begin{array}{c}\text { Grain } \\
\text { yield }\end{array}$} & \multirow{2}{*}{$\begin{array}{c}\text { Straw/Stalk/ } \\
\text { Stover yield } \\
{\left(\mathrm{kg} \mathrm{ha}^{-1}\right)}^{-1}\end{array}$} & \multicolumn{2}{|c|}{ Kharif } & \multicolumn{2}{|c|}{$R a b i$} & & & \\
\hline & & & & & & Grain & Straw & Grain & Straw & Kharif & Rabi & System \\
\hline T1 & Maize - Sunflower (Check) & 4812 & 8952 & 752 & 976 & 4812 & 656 & 2176 & 0 & 5468 & 2176 & 7644 \\
\hline \multirow[t]{2}{*}{ T2 } & \multirow[t]{2}{*}{ Pear millet + Soybean $(3: 2)$ - Potato } & 1037 & 1287 & \multirow[t]{2}{*}{2649} & \multirow[t]{2}{*}{847} & \multirow[t]{2}{*}{1773} & \multirow[t]{2}{*}{102} & \multirow[t]{2}{*}{4852} & \multirow[t]{2}{*}{0} & \multirow[t]{2}{*}{1875} & \multirow[t]{2}{*}{4852} & \multirow[t]{2}{*}{6727} \\
\hline & & 389 & 442 & & & & & & & & & \\
\hline T3 & Maize - Groundnut & 5187 & 8721 & 882 & 1081 & 5187 & 639 & 2727 & 245 & 5826 & 2972 & 8798 \\
\hline \multirow[t]{2}{*}{ T4 } & \multirow{2}{*}{$\begin{array}{l}\text { Pear millet + Soybean }(3: 2)- \\
\text { Sunflower + Groundnut }(2: 3)\end{array}$} & 887 & 1153 & 323 & 484 & \multirow[t]{2}{*}{1650} & \multirow[t]{2}{*}{92} & \multirow[t]{2}{*}{3111} & \multirow[t]{2}{*}{202} & \multirow[t]{2}{*}{1742} & \multirow[t]{2}{*}{3313} & 5055 \\
\hline & & 401 & 391 & 704 & 892 & & & & & & & \\
\hline T5 & Maize + Soybean (2:3) - Potato & 3562 & 6982 & 2573 & 801 & 4414 & 520 & 4712 & 0 & 4934 & 4712 & 9646 \\
\hline & & 435 & 463 & & & & & & & & & \\
\hline T6 & Bt cotton + Soybean $(1: 3)-$ Sesame + & 2150 & 3439 & 185 & 409 & 7507 & 9 & 2477 & 162 & 7516 & 2639 & 10155 \\
\hline & Groundnut & 487 & 512 & 582 & 714 & & & & & & & \\
\hline T7 & Maize (Flat) + Soybean (Raised Bed) - & 3721 & 7234 & 952 & 1921 & 4524 & 538 & 3616 & 80 & 5062 & 3696 & 8758 \\
\hline & Castor $(\mathrm{F})+$ Green gram $(\mathrm{RB})(2: 3)$ & 410 & 431 & 307 & 527 & & & & & & & \\
\hline T8 & Bt cotton + Greengram (1:3) - Pearl & 2031 & 4732 & 1308 & 2531 & 7375 & 100 & 1274 & 193 & 7474 & 1468 & 8942 \\
\hline & millet & 337 & 680 & & & & & & & & & \\
\hline T9 & Soybean - Potato & 1252 & 1132 & 2872 & 957 & 2454 & 21 & 5260 & 0 & 2474 & 5260 & 7734 \\
\hline T10 & Bt cotton + Green gram (1:2) -Sesame & 1987 & 4710 & 356 & 894 & 6878 & 66 & 1304 & 0 & 6945 & 1304 & 8249 \\
\hline & & 234 & 452 & & & & & & & & & \\
\hline T11 & Pearl millet - Groundnut & 1302 & 1532 & 934 & 1056 & 1269 & 112 & 2888 & 239 & 1381 & 3127 & 4508 \\
\hline T12 & Bt Cotton+ Green gram (1:2) -Maize & 1905 & 3975 & 14837 & 11624 & 6572 & 58 & 4322 & 852 & 6630 & 5173 & 11803 \\
\hline & for green cobs & 218 & 397 & & & & & & & & & \\
\hline & S Em \pm & & & & & & & & & 270.9 & 154.8 & \\
\hline & CD $(0.05)$ & & & & & & & & & 799.7 & 456.9 & \\
\hline & CV (\%) & & & & & & & & & 9.97 & 7.91 & \\
\hline
\end{tabular}

Kharif: Sale price for Grain $\left(\mathrm{kg}^{-1}\right)$ : Maize $=$ Rs 13.65, Pearl millet $=$ Rs 13.30, Soybean $=$ Rs 27.75, Bt Cotton $=$ Rs 41.60, Greengram $=$ Rs 52.25 Sunflower $=$ Rs 39.50, Groundnut $=$ Rs 42.20, Sesame $=$ Rs 50.00, Castor $=$ Rs. 35.00, Potato $=$ Rs 25.00 Sale price for stover $\left(\mathrm{kg}^{-1}\right):$ Maize $=$ Rs 1.00, Pearl millet $=$ Rs 1.00, Soy4bean $=0.25$, Greengram $=$ Rs 2.00 , Groundnut Rs 3.00

Rabi: Sale price for grain (kg-1) : Maize (green cobs) $=$ Rs 5/kg, Pearl millet $=$ Rs 13.30, Soybean $=$ Rs 27.75, Bt Cotton $=$ Rs 41.60, Sunflower $=$ Rs 39.50, Groundnut $=$ Rs 42.20, Sesame $=$ Rs 50.00, Castor $=$ Rs 35.00, Potato $=$ Rs $25.00 ;$ Sale price for stover $(\mathrm{kg}-1):$ Maize $=$ Rs 1.00 , Pearl millet $=$ Rs 1.00 , Greengram Rs 2.00, Groundnut Rs 3.00 
Table.2 Economics of crops under the bio-intensive complementary cropping systems

\begin{tabular}{|c|c|c|c|c|c|c|c|c|c|c|c|}
\hline & Treatment & & Khar & & & & $R a b i$ & & & & tem \\
\hline & Kharif-Rabi & Cost of & & Net $r$ & urns & & & Net $r$ & urns & Net & turns \\
\hline & & $\begin{array}{c}\text { cultivation } \\
\left(\text { Rs. ha }{ }^{-1}\right)\end{array}$ & $\begin{array}{c}\text { returns } \\
\left(\text { Rs. ha }^{-1}\right)\end{array}$ & Rs. ha $^{-1}$ & Rs. $\mathbf{R e}^{-}$ & $\begin{array}{c}\text { cultivation } \\
\left(\text { Rs. ha }^{-1}\right)\end{array}$ & $\begin{array}{l}\text { returns } \\
\text { (Rs. ha } \\
\left.{ }_{1}^{-}\right)\end{array}$ & Rs. ha $^{-}$ & $\begin{array}{l}\mathbf{R e}^{-1} \\
\mathbf{R e}^{-1}\end{array}$ & $\underset{1}{\text { Rs. ha" }}$ & Rs. $\operatorname{Re}^{-1}$ \\
\hline T1 & Maize - Sunflower (Check) & 43065 & 74636 & 31571 & 0.73 & 26893 & 29704 & 2811 & 0.10 & 34382 & 0.49 \\
\hline $\mathbf{T 2}$ & Pear millet + Soybean $(3: 2)$ - Potato & 20460 & 25595 & 5135 & 0.25 & 58053 & 66225 & 8172 & 0.14 & 13307 & 0.17 \\
\hline T3 & Maize - Groundnut & 43065 & 79524 & 36459 & 0.85 & 35150 & 40561 & 5411 & 0.15 & 41870 & 0.54 \\
\hline T4 & $\begin{array}{l}\text { Pear millet }+ \text { Soybean }(3: 2)-\text { Sunflower }+ \\
\text { Groundnut }(2: 3)\end{array}$ & 20460 & 23775 & 3315 & 0.16 & 33168 & 45224 & 12056 & 0.36 & 15371 & 0.29 \\
\hline T5 & Maize + Soybean (2:3) - Potato & 47390 & 67355 & 19965 & 0.42 & 58053 & 64325 & 6272 & 0.11 & 26237 & 0.25 \\
\hline T6 & $\begin{array}{l}\text { Bt cotton }+ \text { Soybean }(1: 3)-\text { Sesame }+ \\
\text { Groundnut }\end{array}$ & 56250 & 102595 & 46345 & 0.82 & 27886 & 36017 & 8131 & 0.29 & 54476 & 0.65 \\
\hline T7 & $\begin{array}{l}\text { Maize (Flat) + Soybean }(\mathrm{RB})-\text { Castor }(\mathrm{F})+ \\
\text { Green gram }(\mathrm{RB})(2: 3)\end{array}$ & 49390 & 69101 & 19711 & 0.40 & 27793 & 50447 & 22654 & 0.82 & 42365 & 0.55 \\
\hline T8 & Bt cotton + Greengram (1:3) - Pearl millet & 53350 & 102206 & 48676 & 0.91 & 17005 & 20034 & 3029 & 0.18 & 51705 & 0.73 \\
\hline T9 & Soybean - Potato & 27560 & 33774 & 6214 & 0.23 & 58053 & 71800 & 13747 & 0.24 & 19961 & 0.23 \\
\hline T10 & Bt cotton + Green gram (1:2) -Sesame & 51370 & 94795 & 43425 & 0.85 & 15995 & 17800 & 1805 & 0.11 & 45230 & 0.67 \\
\hline T11 & Pearl millet - Groundnut & 16140 & 18849 & 2709 & 0.17 & 35150 & 42678 & 7528 & 0.21 & 10237 & 0.20 \\
\hline T12 & $\begin{array}{l}\text { Bt Cotton+ Green gram (1:2) -Maize for } \\
\text { green cobs }\end{array}$ & 51370 & 90506 & 39136 & 0.76 & 30930 & 70614 & 39684 & 1.28 & 78820 & 0.96 \\
\hline
\end{tabular}


Table.3 Total Nutrient uptake (Nitrogen, Phosphorus and Potassium) by different cropping systems

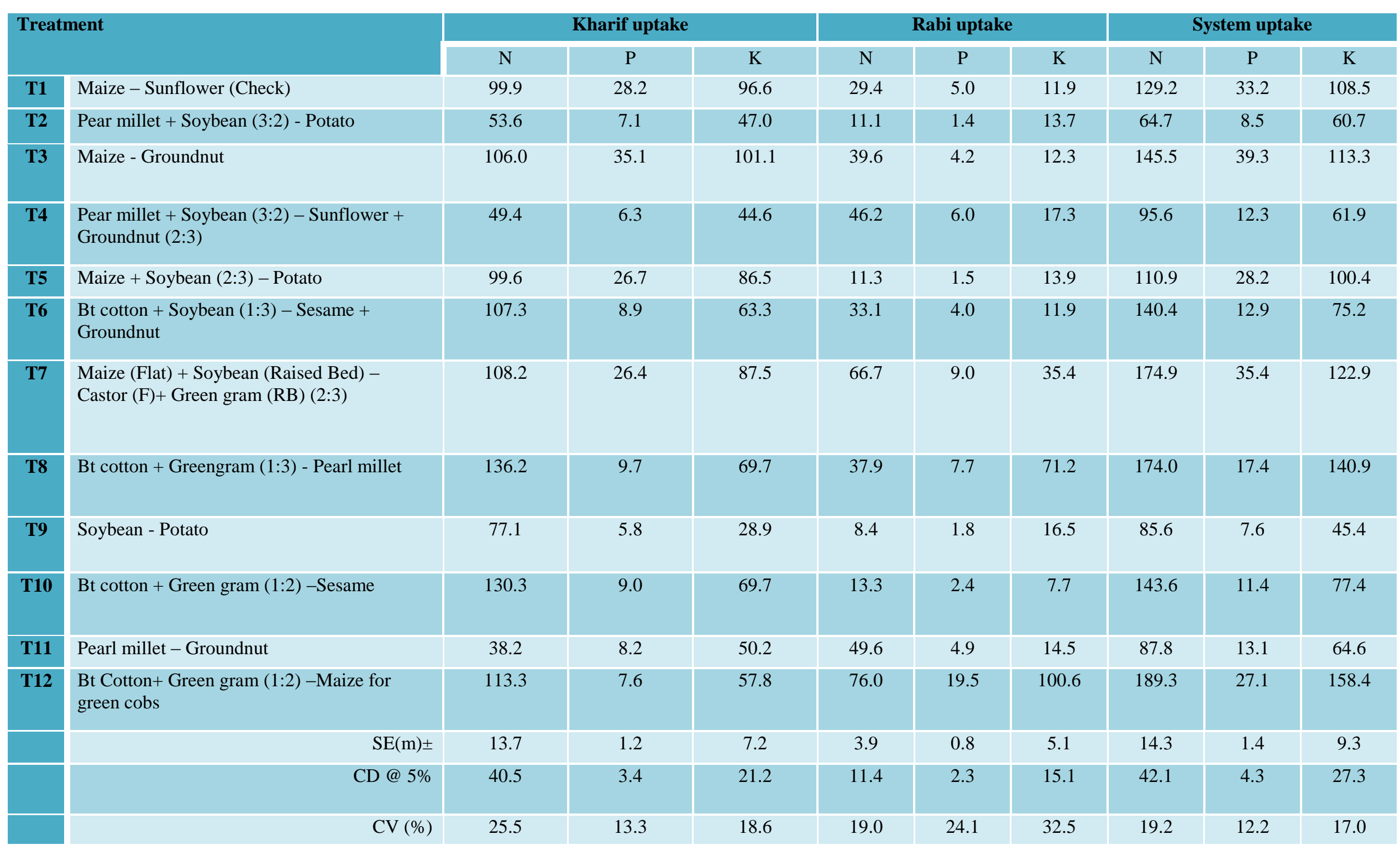


Table.4 Soil fertility status at the end of kharif and rabi under the bio-intensive complementary cropping systems

\begin{tabular}{|c|c|c|c|c|c|c|c|c|c|c|c|c|c|}
\hline \multirow[t]{3}{*}{ Trt } & \multirow[t]{3}{*}{ Cropping sequence } & \multicolumn{6}{|c|}{ Kharif } & \multicolumn{6}{|c|}{ Rabi } \\
\hline & & \multirow[t]{2}{*}{ pH } & \multirow[t]{2}{*}{$\begin{array}{c}\mathrm{EC} \\
\left(\mathrm{dS} \mathrm{m}^{-1}\right)\end{array}$} & \multirow[t]{2}{*}{$\begin{array}{l}\mathrm{OC} \\
(\%)\end{array}$} & \multicolumn{3}{|c|}{$\begin{array}{l}\text { Avail. Nutrients } \\
\qquad\left(\mathrm{kg} \mathrm{ha}^{-1}\right)\end{array}$} & \multirow[t]{2}{*}{ pH } & \multirow[t]{2}{*}{$\begin{array}{c}\mathrm{EC} \\
\left(\mathrm{dS} \mathrm{m}^{-1}\right)\end{array}$} & \multirow[t]{2}{*}{$\begin{array}{l}\mathrm{OC} \\
(\%)\end{array}$} & \multicolumn{3}{|c|}{$\begin{array}{l}\text { Avail. Nutrients } \\
\qquad\left(\mathrm{kg} \mathrm{ha}^{-1}\right)\end{array}$} \\
\hline & & & & & $\mathbf{N}$ & $\mathbf{P}$ & $\mathbf{K}$ & & & & $\mathbf{N}$ & $\mathbf{P}$ & $\mathbf{K}$ \\
\hline & Initial & 8.50 & 0.38 & 0.59 & & 30.7 & 274.8 & & & & & & \\
\hline T1 & Maize - Sunflower (C) & 8.06 & 0.26 & 0.51 & 185.4 & 34.9 & 247.3 & 8.51 & 0.26 & 0.56 & 189.3 & 28.3 & 242.7 \\
\hline $\mathbf{T 2}$ & Pear millet + Soybean $(3: 2)-$ Potato & 8.04 & 0.24 & 0.53 & 215.5 & 38.0 & 248.8 & 8.36 & 0.28 & 0.59 & 209.3 & 38.1 & 257.0 \\
\hline T3 & Maize - Groundnut & 8.05 & 0.26 & 0.57 & 227.9 & 39.5 & 255.9 & 8.53 & 0.28 & 0.44 & 207.7 & 34.2 & 261.8 \\
\hline T4 & $\begin{array}{l}\text { Pear millet + Soybean }(3: 2)- \\
\text { Sunflower + Groundnut }(2: 3)\end{array}$ & 8.20 & 0.22 & 0.56 & 240.6 & 37.2 & 232.3 & 8.34 & 0.33 & 0.55 & 210.8 & 38.6 & 258.9 \\
\hline T5 & Maize + Soybean (2:3) - Potato & 8.05 & 0.28 & 0.52 & 238.3 & 35.8 & 223.0 & 8.45 & 0.23 & 0.53 & 203.1 & 30.8 & 257.0 \\
\hline T6 & $\begin{array}{l}\text { Bt cotton }+ \text { Soybean }(1: 3)-\text { Sesame }+ \\
\text { Groundnut }\end{array}$ & 8.20 & 0.33 & 0.61 & 206.1 & 27.4 & 228.6 & 8.36 & 0.32 & 0.58 & 223.3 & 33.1 & 286.3 \\
\hline T7 & $\begin{array}{l}\text { Maize (Flat) + Soybean (Raised Bed) } \\
\text { - Castor (F)+ Gr. gram (RB) }(2: 3)\end{array}$ & 8.37 & 0.25 & 0.52 & 231.0 & 37.1 & 244.3 & 8.69 & 0.35 & 0.54 & 221.3 & 30.5 & 254.5 \\
\hline T8 & $\begin{array}{l}\text { Bt cotton + Greengram (1:3) - Pearl } \\
\text { millet }\end{array}$ & 7.99 & 0.32 & 0.55 & 211.2 & 27.5 & 240.6 & 8.39 & 0.34 & 0.57 & 194.1 & 32.4 & 237.2 \\
\hline T9 & Soybean - Potato & 8.20 & 0.22 & 0.51 & 230.4 & 41.8 & 281.6 & 8.30 & 0.35 & 0.55 & 196.4 & 34.5 & 287.8 \\
\hline T10 & Bt cotton + Green gram (1:2) -Sesame & 8.03 & 0.31 & 0.56 & 222.9 & 33.7 & 232.7 & 8.64 & 0.35 & 0.47 & 203.9 & 28.2 & 270.6 \\
\hline T11 & Pearl millet - Groundnut & 8.08 & 0.28 & 0.59 & 227.0 & 37.2 & 247.7 & 8.45 & 0.31 & 0.50 & 200.1 & 29.0 & 277.2 \\
\hline \multirow[t]{3}{*}{ T12 } & $\begin{array}{l}\text { Bt Cotton+ Green gram }(1: 2) \text {-Maize } \\
\text { for green cobs }\end{array}$ & 8.31 & 0.32 & 0.58 & 191.9 & 33.0 & 260.7 & 8.36 & 0.24 & 0.59 & 191.4 & 34.9 & 265.0 \\
\hline & $\mathrm{SEm} \pm$ & 0.16 & 0.05 & 0.06 & 14.8 & 3.0 & 17.6 & 0.10 & 0.04 & 0.03 & 9.2 & 4.1 & 13.8 \\
\hline & CD (at 5\%) & NS & NS & NS & NS & 8.8 & NS & NS & NS & NS & NS & NS & NS \\
\hline
\end{tabular}


The high profitability of potato as a cash crop has made it an economically viable enterprise for the small and marginal farmers and has contributed to increasing equity among farmers (Gulati et al., 2007). The lowest MEY was noticed with sesame crop $(1304 \mathrm{~kg}$ ha $^{-1}$ ) grown after $B t$ cotton intercropped with greengram at 1:2 row ratio. However maize grown for green cobs recorded significantly higher net returns (Rs $39684 \mathrm{ha}^{-1}$ ) over other crops.

In terms of system productivity, Bt Cotton + green gram (1:2) - maize for green cobs system recorded highest MEY (11803 kg ha $\left.{ }^{-1}\right)$ with Rs $78820 \mathrm{ha}^{-1}$ of net returns, $0.96 \mathrm{BC}$ ratio followed by $B t$ cotton + Soybean (1:3) Sesame + Groundnut which registered 10155 $\mathrm{kg} \mathrm{ha}^{-1}$ of MEY, net returns of Rs $54476 \mathrm{ha}^{-1}$ and $0.65 \mathrm{BC}$ ratio. The lowest MEY was recorded with pearlmillet - groundnut cropping system $\left(4508 \mathrm{~kg} \mathrm{ha}^{-1}\right)$ with net returns of Rs $10237 \mathrm{ha}^{-1}$. In two year cottonlegume-corn rotation, an yield increase to the tune of 11 per cent was recorded as compared to continuous cotton grown without legumes (Sankaranarayanan et al., 2010). Six Bt cotton based double cropping systems viz., two millets, two pulses and two oilseed crops were evaluated to identify the most profitable, productive and sustainable system. Amongst them, Bt cotton - maize recorded the highest seed cotton equivalent yield (CICR, 2009-10). Banik et al., (2009) also reported that cereallegume intercropping systems were superior to mono cropping.

\section{Nutrient uptake}

Nutrient uptake by various crops and cropping systems varied significantly during kharif 2016 (Table 3). All the systems that have maize or Bt cotton as component crop in the cropping system were found to be on par. Bt cotton + greengram (1:3) cropping system removed significantly higher quantities of nitrogen (136.2 $\mathrm{kg} \mathrm{ha}^{-1}$ and $130.3 \mathrm{~kg} \mathrm{ha}^{-1}$ ) and was closely followed by maize (Flat) + soybean (Raised Bed) system (108.2 kg ha ${ }^{-1}$ ) and maize + soybean $(2: 3)\left(107.3 \mathrm{~kg} \mathrm{ha}^{-1}\right)$ and maize $\left(106.0 \mathrm{~kg} \mathrm{ha}^{-1}\right)$ and were on par with nitrogen removal of cotton based systems. Pearlmillet removed lowest quantities of nitrogen $\left(38.2 \mathrm{~kg} \mathrm{ha}^{-1}\right)$ than all other crops and cropping systems tested. While removal of phosphorus was significantly more by maize $\left(35.1 \mathrm{~kg} \mathrm{ha}^{-1}\right)$ and maize + soybean $(2: 3)$ system $\left(26.7 \mathrm{~kg} \mathrm{ha}^{-1}\right)$ and maize (Flat) + soybean (Raised Bed) system (26.4 $\mathrm{kg} \mathrm{ha}^{-1}$ ) when compared to all other crops and cropping systems (5.8 to $\left.9.0 \mathrm{~kg} \mathrm{ha}{ }^{-1}\right)$. Potassium removal was also significantly more by maize $\left(101.1 \mathrm{~kg} \mathrm{ha}^{-1}\right)$ and maize + soybean (2:3) system (86.57 $\left.\mathrm{kg} \mathrm{ha}^{-1}\right)$ and maize (Flat) + soybean (Raised Bed) system $\left(87.5 \mathrm{~kg} \mathrm{ha}^{-1}\right)$ and was lowest by soybean (28.9 kg ha ${ }^{-1}$ ). During rabi 2016-17, among the crops, maize cultivated for green cobs removed significantly higher nitrogen (76.6 $\left.\mathrm{kg} \mathrm{ha}{ }^{-1}\right)$, phosphorus (19.5 $\mathrm{kg} \mathrm{ha}^{-1}$ ) and potassium $\left(100.6 \mathrm{~kg} \mathrm{ha}^{-1}\right)$ than all other crops and cropping systems tested and potato crop removed the lowest quantities of nitrogen $(8.4$ $\left.\mathrm{kg} \mathrm{ha}^{-1}\right)$, and phosphorus (1.4 $\left.\mathrm{kg} \mathrm{ha}^{-1}\right)$, while potassium removal was lowest by sesame (7.7 $\left.\mathrm{kg} \mathrm{ha}^{-1}\right)$. Bt Cotton + green gram (1:2)-maize for green cobs system removed significantly higher nitrogen (189.2 $\left.\mathrm{kg} \mathrm{ha}^{-1}\right)$ and was closely followed by and on par with maize (Flat) + soybean (Raised Bed) - castor $(\mathrm{F})+$ green gram (RB) $(2: 3)$ system $\left(174.9 \mathrm{~kg} \mathrm{ha}^{-1}\right)$ and Bt cotton + green gram $(1: 3)$-pearlmillet $\left(174.0 \mathrm{~kg} \mathrm{ha}^{-1}\right)$ while pear millet + soybean $(3: 2)$ - potato system removed lowest quantities of nitrogen $\left(64.7 \mathrm{~kg} \mathrm{ha}^{-1}\right)$ than all other crops and cropping systems tested. Removal of phosphorus was significantly more by maize based cropping systems viz., maize -groundnut (39.3 kg ha $\left.{ }^{-1}\right)$, maize (Flat) + soybean (Raised Bed) - castor $(\mathrm{F})+$ green gram (RB) (2:3) system $\left(35.4 \mathrm{~kg} \mathrm{ha}^{-1}\right)$, maize - sunflower $\left(33.2 \mathrm{~kg} \mathrm{ha}^{-1}\right)$, maize + soybean 
(2:3) - potato system $\left(28.2 \mathrm{~kg} \mathrm{ha}^{-1}\right)$, Bt Cotton + green gram (1:2)-maize for green cobs system $\left(27.1 \mathrm{~kg} \mathrm{ha}^{-1}\right)$ and when compared to all other cropping systems and removal was lowest by soybean-potato $\left(7.6 \mathrm{~kg} \mathrm{ha}^{-1}\right)$. Potassium removal was significantly different in all the systems, and was more by maize and Bt cotton based systems with significantly highest removal in $\mathrm{Bt}$ Cotton+ green gram $(1: 2)$-maize for green cobs system $(158.4 \mathrm{~kg}$ $\mathrm{ha}^{-1}$ ) followed by on par removal with $\mathrm{Bt}$ Cotton+ green gram $(1: 2)+$ pearlmillet system $\left(140.9 \mathrm{~kg} \mathrm{ha}^{-1}\right)$ while it was lowest by soybean - potato cropping sequence $(45.4 \mathrm{~kg}$ $\left.\mathrm{ha}^{-1}\right)$.

\section{Soil Fertility}

The soil $\mathrm{pH}, \mathrm{EC}, \mathrm{OC}$ and available nutrient status (nitrogen and potassium) values after the sequences did not differ significantly from the initial values (Table 4). However, availability of phosphorus was found to be influenced by various cropping systems. Higher available phosphorus was recorded after soybean crop during kharif in soybeanpotato cropping system, but at the end of crop sequence no significant changes were recorded in soil fertility. It can be inferred that alternate systems gave better results in terms of productivity in comparison to prevailing maize-sunflower system in Southern Telangana Zone. Cotton + green gram (1:2) maize for green cobs system recorded highest MEY (11803 kg ha ${ }^{-1}$ ) with Rs 78,820 ha ${ }^{-1}$ net returns followed by $B t$ cotton + soybean $(1: 3)$ - sesame + groundnut (T6) system (10155 kg $\left.\mathrm{ha}^{-1}\right)$.

\section{References}

Banik, P. and R.C. Sharma. 2009. Yield and resource use efficiency in baby corn legume intercropping system in the eastern plateau of India. J. Sustainable Agric., 33: 379 - 395.

CICR. 2010. Annual report, 2009-2010. Central Institute for Cotton Research, Nagpur.

Gulati A, Minot N, Delgado C, Bora S. 2007. Growth in high-value agriculture in Asia and the emergence of vertical links with farmers. In: Swnnen, JFM (ed). Global Supply Chains, Standards and the Poor. CABI Publ, Oxford.

Hedge, D.M., Tiwari, P.S., and Rai, M. 2003. Crop diversification in Indian Agriculture. Agricultural Situation in India, 60(5): 255-272.

Jackson, M. L. 1973. Soil chemical analysis. Prentice Hall of India, Pvt. Ltd. New Delhi. Physiology. 10: 400-403.

Sankaranarayanan, K., Praharaj, C.S., Nalayini, P., Bandyopadhya, K.K., and Gopalakrishnan, N. 2010. Legume as companion crop for cotton. J. Cotton Res. Dev. 24 (1): 115-126.

Sree Rekha, M. and Dhurua, S. 2010. Fertiliser management in cotton + soybean (1:2) intercropping system under rainfed conditions. J. Cotton Res. Dev. 24 (1): 67-70.

Yadav, J.S.P. 2002. Agricultural resource management in India: The challenges. Journal of Agricultural Water Management, 1(1): 61-69.

\section{How to cite this article:}

Pragathi Kumari, Ch., S. Sridevi and Goverdhan, M. 2018. Profitable Cropping Systems for Southern Telangana Zone of Telangana State, India. Int.J.Curr.Microbiol.App.Sci. 7(01): 25182525. doi: https://doi.org/10.20546/ijcmas.2018.701.302 